\title{
OS SÓCIO-ANTROPO-LOGOS E OS ANIMAIS. REFLEXÕES DE UM HISTORIADOR PARA UMA REAPROXIMAÇÃO DAS CIÊNCIAS
}

\author{
Éric Baratay ${ }^{1}$
}

Que o leitor não se engane! A minha intervenção não tem como objetivo julgar a validade do trabalho de outras disciplinas diferentes da minha, algo que eu não poderia e não gostaria de fazer. Trata-se, à convite dos organizadores desta edição especial ${ }^{2}$, da apresentação do relato de um leitor externo sobre as pesquisas sociológicas e antropológicas referentes ao animal, sobre o interesse da sua evolução para o historiador, sobre a utilidade dos conceitos propostos e dos dados fornecidos para a sua própria pesquisa, mas também sobre o que essas ciências humanas poderiam buscar na produção histórica, e o que poderia ser feito em conjunto, também com outras ciências. Retomando e estendendo um artigo de 1997 sobre a historiografia do animal, onde abordei a questão do uso histórico das ciências humanas ${ }^{3}$. Remeto o leitor interessado a este artigo de modo à reservar as seguintes proposições sobre as pesquisas da última década.

\section{As abordagens críticas estruturalistas}

Em primeiro lugar, devo confessar que como historiador me sinto mais confortável e em sintonia com a recente produção acadêmica, ao invés daquela de 1980 ou 1990, por duas razões.

A primeira situa-se no quase desaparecimento de uma literatura de sarcasmo ou de denúncia, que florescia quando alguns pensavam no assunto sem interesse, e quando outros criticavam as práticas contemporâneas, notadamente as urbanas, em termos de antiguidade ou de ruralidades erigidas como modelo em relação ao animal, o que dificultava uma análise original, confirmando-se ilusória, logo, os historiadores têm apontado que a ideia de um rural eternamente cheio de animais está por corrigir: a

\footnotetext{
${ }^{1}$ Universidade Lyon III, França.

${ }^{2} \mathrm{O}$ autor refere-se ao dossier da revista Societés $\left(2010 / 2 \quad\left(\mathrm{n}^{\circ}\right.\right.$ 108), intitulado Relations Antropozoologiques, organizado por Emmanuel Gouabault e Jérôme Michalon.

3 «Un champ pour l'histoire : l'animal », Cahiers d'histoire, 1997, 3-4: 409-442, em colaboração com J.L. Mayaud que redigiu: 424-426 consagradas à história da produção animal.
} 
disseminação do cão pastor no campo, por exemplo, começa somente a partir do século XVIII, paralelamente com a do cão de companhia nas cidades ${ }^{4}$.

A segunda razão vem de um ínfimo entusiasmo para análises estruturais ou sistêmicas que são intelectualmente estimulantes, como a maravilhosa análise de Philippe Descola sobre as relações com a natureza ${ }^{5}$, mas que têm o inconveniente, a meu ver, de deixar o historiador à beira da estrada, desnorteado e impotente, excluindo a história da sua reflexão na busca de invariantes humanas, uma pesquisa que não é inerente à antropologia, porque a noção de evolução estava no centro das suas reflexões no século XIX, mas se impôs no século XX para combater o racismo científico e, sobretudo, para copiar as ciências duras, para encontrar leis da natureza humana, pensadas como uma entidade fixa.

No entanto, o estudo de um objeto requer uma hora ou outra, a conciliação das diferentes abordagens disciplinares se não quisermos privilegiar os discursos de investigação em detrimento de conhecimento do assunto, necessariamente complexo e plural. Mas não é fácil conciliar uma análise que reúna dados dispersos no tempo em um sistema atemporal, cuja explicação referia-se a uma psicologia humana invariável, de uma outra que chama a atenção ao contrário pela flutuação das representações, invocando uma evolução das psicologias. Tomemos o exemplo concreto do urso: como conciliar o belo trabalho de antropologia simbólica de Sophie Bobbé ao igualmente notável, de Michel Pastoureau6? Ninguém se dispôs até o momento e a dificuldade encorajou cada um a ficar em casa ignorando os caminhos dos outros.

\section{Das leituras culturalistas ao estudo dos coletivos humano-animais}

A empreitada é mais fácil e sua utilidade mais imediata a partir de outros modos de leitura. Assim, a abordagem culturalista centrada sobre a análise das representações e das práticas contemporâneas, como é conduzida por exemplo, por Sergio Della Bernardina ou Marie Cegarra ${ }^{7}$; uma abordagem que pode também

\footnotetext{
${ }^{4}$ Voir R. Béteille, Le Chien, Paris, PUF, 1997 ; É. Baratay, La Société des animaux, de la Révolution à la Libération, Paris, La Martinière, 2008. A gente encontra ainda uma tendência à denúncia em C. TourreMalen, Femmes à cheval, la féminisation des sports et des loisirs équestres: une avancée?, Paris, Belin, 2006.

${ }^{5}$ Par-delà nature et culture, Paris, Gallimard, 2005.

${ }^{6}$ S. Bobbé, L'Ours et le loup. Essai d'anthropologie symbolique, Paris: MSH, 2002; M. Pastoureau, L'Ours, histoire d'un roi déchu, Paris: Seuil, 2007.

${ }^{7}$ S. Dalla Bernardina, L'Utopie de la nature, chasseurs, écologistes et touristes, Paris, Imago, 1998, et
} 
desenvolver uma reflexão sistêmica, tal qual a de Frederick Saumade sobre as touradas europeias $^{8}$, mas as limitando no tempo presente e incorporando a ideia de evolução, muitas vezes sob a influência de Norbert Elias, que colocora as lentas modificações sóciopisíquicas no centro de sua obra. Ora, esta operarando uma síntese magistral entre a sociologia e a história, influenciou particularmente os historiadores, como evidenciam os relevantes trabalhos de Alain Corbin ${ }^{9}$, ao passo que a abordagem culturalista também se impôs com a história cultural, que domina a cena histórica há vinte anos, a tal ponto que a maioria dos trabalhos sobre o animal partem desta leitura ${ }^{10}$. A proximidade e, portanto, a familiaridade da abordagem são tão evidentes com o estudo dos conflitos contemporâneos, com as representações ou com as práticas, que se tornou uma das principais vias de análise das ciências humanas, como ilustra a obra de Isabelle Mauz sobre os lobos alpinos, ou a recente edição da Ethnologie française sobre "os animais da discórdia"11.

O interesse é particularmente tão mais forte para o historiador quanto os conflitos que envolvem circulações, adaptações, portanto uma dinâmica, uma evolução, uma vez que este tema é também objeto de obras históricas em número crescente $^{12}$. Enfim, o historiador deveria dar mais importância ao surgimento recente do estudo dos "coletivos" homens-animais, ou das "comunidades híbridas", inicialmente proposto por Bruno Latour, Dominique Lestel e Vinciane Despret ${ }^{13}$. A abordagem tem o grande interesse de integrar o ser animal na pesquisa, uma vez que o mesmo está ausente dos trabalhos quando adentramos nas leituras anteriores, sendo tratado apenas como um objeto que permite evocar as práticas humanas.

Esta abordagem também tem a vantagem de sair de uma visão demasiadamente simples, maniqueísta, onde o homem pensante, agente, deixaria suas

L'Éloquence des bêtes: quand l'homme parle des animaux, Paris, Métaillé, 2006 ; M. Cegarra, L'animal inventé. Ethnographie d'un bestiaire familier, Paris, L'Harmat- tan, 2000. Consultar também sobre os zoos, Techniques et culture, 50, 2008.

${ }^{8}$ Les Tauromachies européennes. La forme et l'histoire, une approche anthropologique, Paris: CTHS, 1998.

${ }^{9}$ A partir de Le Miasme et la jonquille. L'odorat et l'imaginaire social, XVIIIe-XIXe siècle, Paris, Aubier, 1982. Norbert Elias a d'ailleurs abordé l'histoire de l'animal avec la chasse au renard: Sport et civilisation, la violence maîtrisée (1986), Paris, Fayard, 1994.

${ }^{10}$ Um exemplo recente: « Figures animales », Sociétés et représentations, 27, 2009.

${ }^{11}$ Gens, cornes et crocs, Versailles, INRA, 2005; Ethnologie française, 39, 2009, 1.

${ }^{12}$ Por exemplo: J.L. Mayaud, 150 ans d'excellence agricole en France. Histoire du con-cours général agricole, Paris: Belfond, 1991; N. de Blomac, Voyer d'Argenson et le cheval des Lumières, Paris: Belin, 2004.

${ }^{13}$ B. Latour, Nous n'avons jamais été modernes, Paris: La Découverte, 1991; D. Lestel, L'Animal singulier, Paris: Seuil, 2004; V. Despret, Bêtes et hommes, Paris: Gallimard, 2007. 
concepções e práticas sobre um animal passivo e submiço, e que ainda está presente no centro das atenções entre os pesquisadores das diversas ciências humanas, marcadas pela ideologia do domínio da natureza, fortemente reativada entre as décadas de 1930 e 1980. Colocando em evidência as ações dos animais, as interações com os homens e as reações destes, esta abordagem se revela frutífera como mostram os trabalhos de Albert Piette sobre o "fato socio-animal", de Dominique Guillo, propondo uma sociologia interespecífica sobre o cão, de Catherine Rémy pelo fim das feras, ou de Isabelle Mauz $^{14}$. Ora, as interações também supõem um processo dinâmico que pode permitir a reintrodução da história nas ciências humanas, ao mesmo tempo em que os historiadores também descobrem o interesse pelos "coletivos" ${ }^{15}$.

\section{Nutrir-se uns dos outros}

Situando-se, assim, no estudo dos processos dinâmicos, a sociologia, a antropologia e a história podem contribuir bastante, as primeiras fornecendo a ultima conceitos e hipóteses, mais fáceis de construir sobre atualidade viva que sobre um passado morto, quebrado, disperso nos arquivos, que o historiador deve usar para melhor interrogar e ler seus documentos, para trazer à tona os aspectos que teriam sido difíceis ver sem eles. Assim é o caso, há muito tempo, do processo de civilização de Elias, mas deveria ser também o caso para o animal, da sociologia dos não-humanos, da ligação dos coletivos, do fato socio-animal, etc. Por outro lado, a história deveria permitir a comprovação da validade temporal destes conceitos, a nuance do vigor das análises, o delineamento da gênese e a evolução dos fatos contemporâneos, a construção de sínteses diacro-sincrônicas sobre as representações, as práticas, os conflitos, as interações, etc.

Que o leitor me perdoe, mas não é necessariamente o caso ao ler as bibliografias de estudos sociológicos e antropológicos, que citam poucos os trabalhos, agora numerosos, sobre a história dos animais. Parece que depois de ter renunciado com

\footnotetext{
${ }^{14}$ A. Piette, «Entre 1'homme et le chien. Pour une ethnographie du fait socio-animal », Socioanthropologie, 11, 2002: 87-104; D. Guillo, Des Chiens et des humains, Paris, Pommier, 2009; C. Rémy, La Fin des bêtes. Une ethnographie de la mise à mort des ani- maux, Paris, Economica, 2009; I. Mauz, Les Collectifs et leurs natures, dossier d'HDR, Université de Saint-Étienne, 2008.

15 J.M. Moriceau, Histoire du méchant loup, Paris, Fayard, 2007; É. Baratay, « De l'homme héros à l'animal acteur et retour », Histoire des Alpes, 15, 2010.
} 
frequência a dissolução da história em estruturas atemporais, muitos a ignoram agora, simplesmente separando o passado e o presente. Para não me expor a um julgamento imprudente, reconheço que este é também o caso dos historiadores, e que as considerações sobre a história pelos sociólogos e antropólogos parecem, ainda assim, até mais frequentes do que antes, sem dúvida favorecidas pelo estudo dos processos dinâmicos, buscando traçar a gênese e a evolução, como ilustram as obras de Frederick Saumade, Catherine Tourre-Malen ou Catherine Rémy ${ }^{16}$. O uso da história por eles pode intrigar os historiadores, assim como o uso histórico da sociologia e da antropologia pode espantar os seus especialistas, porque alguns insistem em enfatizar a pluralidade, os movimentos, os caprichos da história e outros sob diretrizes ou genealogias claras de fatos contemporâneos. Não há dúvida de que seria necessário pouco a pouco um melhor entendimento, uma maior colaboração sobre o conteúdo dos conceitos e dos ângulos de análise.

Portanto, uma reconciliação passado-presente é indispensável para um melhor conhecimento plural do sujeito/assunto ${ }^{17}$ animal. Deveria-se acompanhar a clara reaproximação empreendida entre as disciplinas do presente há uma ou duas décadas, não só entre sociólogos e antropólogos, mas também entre geógrafos, que refletem em particular, sobre as delimitações da fronteira humanidade-animalidade, ou sobre o lugar do animal ${ }^{18}$ e os cientistas políticos que observam os conflitos do uso das bestas ${ }^{19}$, a tal ponto que muitas vezes é difícil, ao ler os trabalhos, de distinguir a origem disciplinar dos autores e diferenciar as abordagens, embora muitas edições especiais organizadas por revistas disciplinares reagrupem especialidades diversas, e que antes as abordagens eram específicas de uma disciplina, como por exemplo, segundo as divisões sociais na sociologia, são em menor número ${ }^{20}$. Logo a reaproximação das disciplinas, a mistura dos conceitos, das análises e dos temas, já favorecidos pelo discurso sobre a necessária

\footnotetext{
${ }^{16}$ C. Rémy, «Une mise à mort industrielle « humaine »? L'abattoir ou l'impossible objectivation des animaux», Politix, 64, 2003: 51-73.

${ }^{17}$ Aqui tomamos a liberdade de manter a ambiguidade da palavra sujet que tanto pode significar sujeito quanto assunto, sendo que ambas as possibilidades vão ao encontro do pensamento do autor.

${ }_{18}$ N. Blanc, Les Animaux et la ville, Paris: Odile Jacob, 2000; «La place de l'animal», Espaces et sociétés, 110, 2002; «Protéger la nature, est-ce protéger la société? », Géogra- phie et cultures, 69, 2009.

${ }^{19}$ D. Darbon, La Crise de la chasse en France, Paris, L'Harmattan, 1997; C. Traïni, Les Braconniers de la République. Les conflits autour des représentations de la nature, Paris: PUF, 2003; "La question animale », Politix, 64, 2003.

${ }^{20}$ «Vers une sociologie des relations avec la nature », Revue Française de Sociologie, 48, 2007, 4: 795806; M. Pinçon-Charlot, "La place de l'animal dans la société des XX ${ }^{\mathrm{e}}-\mathrm{XXI}$ e siècles », dans $L a$ Recherche médicale à l'aube du XXIe siècle: recherche médicale et modèle animal, Paris, Elsevier, 2002: 24-28.
} 
pluridisciplinaridade para privilegiar os objetos à serem estudados sobre os campos delimitados disciplinarmente, podem justamente ser reforçados e estendidos especialmente para a história e para as ciências naturais, por uma nova abordagem que provoca rupturas e que será, creio eu, um dos “canteiros de obras” dos próximos anos.

\section{Animal, ator completamente à parte ${ }^{21}$}

$\mathrm{Na}$ verdade, o recente interesse pelos coletivos humanos-animais experimenta no momento um desequilíbrio de atenção e de análise em prol do homem, pensado como verdadeiro ator, em detrimento do animal, concebido como um objeto que age a partir da ação humana, mas não agindo por si só.

Assim, em seu notável artigo, Albert Piette é muito inovador na construção do fato socio-animal e conservador no exemplo concreto da relação homem-cão, que ele considera de bom grado assimétrica e particular no que tange às relações humanas, mas reforçando muito tal aspecto, considera que o cão não é um interlocutor pertinente, que uma parte da interação viria de uma projeção antropomórfica que dotaria o animal de capacidades cognitivas e afetivas indevidas, que o cão fornece apenas uma presença passiva, desprovido de ação ou de desejo estratégico, pois ele baseia esta avaliação em uma literatura etnológica culturalista, construída sobre a ideia de domínio e de dominação humana, e em tratados de educação canina que veiculam a mesma visão, propondo a imposição de uma ordem humana sobre o animal. A relação parece bem diferente, mais rica e, sobretudo, mais equilibrada, especialmente se recorrermos aos questionários dos veterinários junto aos donos, que mostram, por exemplo, que o cão começa as brincadeiras ou pede para passear, e à luz da recente etologia cognitiva, mais disposta a atribuir capacidades aos animais do que as escolas etológicas anteriores.

É preciso procurar essa veia que atribui mais ao animal e que permite ver além, pensando nele e o aceitando como um ator por inteiro, diferente é claro, mas capaz. Deve-se, portanto, estudar direta e precisamente esse ator para, por sua vez, melhor compreender as interações com os homens e as reações com os mesmos.

\footnotetext{
${ }^{21}$ Aqui, novamente é preciso destacar a instigante ambiguidade presente no pensamento do autor, pois tanto o animal como "ator" que detém agências, quanto a reflexão acadêmica sobre o animal, ficaram à parte durante muito tempo no pensamento ocidental.
} 
Essa mudança de olhar sobre o animal é efetuada atualmente na filosofia ${ }^{22}$, nas ciências humanas, quando André Micoud olha para o quotidiano das vacas para compreender o seu destino publicitário, ou quando Marion Vicart estuda de perto o cão de companhia ${ }^{23}$, e na história à partir do antigo apelo de Robert Delort para construir uma história dos animais, não apenas as atitudes dos homens em relação à eles, o qual começa a ser ouvido e gera interesse pelas evoluções das presenças animais, pelas atitudes selvagens, pelas vivências dos domesticados, com base na ideia que a história não é só a ciência dos homens no tempo, mas a ciência dos espaços no tempo ${ }^{24}$.

\section{Pensar as (co)evoluções dos comportamentos}

Aqui, a história pode contribuir bastante para as ciências humanas, mas também para as ciências naturais, em especial sobre a questão da evolução dos comportamentos, que, na minha opinião se tornará central no estudo pluridisciplinar dos animais. Pois, procurando na espessura do tempo, a história pode fornecer dados difíceis a serem estabelecidos ou consolidados quando se trabalha no presente, mesmo que eles possam gerar críticas. Nicolas Lescureux evidenciou a adaptação comportamental dos lobos às evoluções pastoris dos Kirguises, a datar da recente independência do país, quando mostram-se mais audaciosos ao se aproximarem dos vilarejos ${ }^{25}$. Justamente, os historiadores podem mostrar que os lobos poderiam ser antropófagos num período de tempo, ao passo que eram raramente antes, e depois tornaram-se novamente antropogágos; que os cães errantes ou de trabalho do século XIX não se comportavam como os de companhia de hoje em dia; que as vacas deixadas nas planícies antes da especialização leiteira foram arredias com os homens, já que elas eram dóceis quando foram trazidas para os arredores das fazendas e voltavam todos os dias para os estábulos para o trato; que os pitbulls, considerados hoje em dia como cães naturalmente perigosos, eram cães de companhia bastante afetuosos com as crianças nos Estados

\footnotetext{
${ }^{22}$ V. Despret, Penser comme un rat, Versailles, Quae, 2009.

${ }^{23}$ A. Micoud, «Ces bonnes vaches aux yeux si doux», Communications, 74, 2003: 217- 237; M. Vicart, « Faire entrer le chien dans les sciences sociales », Interrogations?, 1, 2005: 131-136.

${ }^{24}$ R. Delort, Les Animaux ont une histoire, Paris, Seuil, 1984 ; J.M. Moriceau, op. cit. ; C. Beck, Les Eaux et forêts en Bourgogne ducale : société et biodiversité, Paris, L'Harmat- tan, 2009 ; É. Baratay, L'Histoire côté animal, autres vécus, autres regards, Paris, Seuil, à paraître, et «Chacun jette son chien. De la fin d'une vie au XIXe siècle », Une bête parmi les hommes: le chien, colloque Valenciennes, 2009, à paraître.

25 « Towards the necessity of a new interactive approach integrating ethnology, ecology and ethology in the study of the relationship between Kyrgyz stockbreeders and wolves », Social, science, information, 45, 2006, 3: 463-478.
} 
Unidos no entreguerras ${ }^{26}$. Estas evoluções são de fato reais e não o fruto da imaginação humana ao sabor de modificações das representações, como se pensava, crendo no animal passivo.

Engajar-se neste caminho requer se questionar sobre as causas dessas mudanças, que podem ser múltiplas, cumulativas, emaranhadas ou sucessivas: evolução dos meios, adaptação dos animais, seleção dos homens, etc. Isso pressupõe a reaproximação da ecologia, para conhecer as configurações dos meios ${ }^{27}$, e da etologia para compreender a aprendizagem. Obviamente, a ideia de evolução dos comportamentos é rejeitada por uma boa parte dos etólogos, sobretudo na França, porque eles aderem à escola lorenziana que fez dos animais seres de comportamentos espontâneos, biologicamente determinados, imutáveis, eliminando assim o tempo e a história, como, ao mesmo tempo, a psicanálise ou o estruturalismo, em conformidade com um dos principais aspectos intelectuais do século XX.

Esta posição é responsável pela controvérsia com Jean-Marc Moriceau sobre a antropofagia dos lobos do Antigo Regime, talvez não tão importante como os documentos podem sugerir, uma vez que os clérigos redatores nem sempre eram bons observadores da natureza, mas claramente ao que parece, o que não foi aceito pelos etnólogos a título de que os lobos atuais não o são (ou não mais). Mas, ainda assim, o tempo estava no centro das reflexões da etologia darwiniana no final do século XIX, e se torna (ou torna-se novamente) com o espaço, um dos parâmetros importantes da etologia de campo, sobretudo na escola cognitiva de origem anglo-saxã, onde já não se hesita em falar de culturas animais entre os grupos dispersos de uma espécie, mas também da evolução cultural de um grupo no mesmo meio, por aprendizagem ou imitação, da adoção da lavagem de batatas dos macacos japoneses à mudança dos cantos entre as baleias jubarte ${ }^{28}$.

O historiador só pode sentir-se próximo a esta perspectiva e, aliás, ele encontra facilmente os traços de uma co-evolução do homem e dos animais, da vaca ao cavalo passando pelo cão, uma ideia que era comum no século XIX e que cientistas, como Yves Christen, agora reativam. A busca de animais atores, agindo realmente em seus

\footnotetext{
${ }^{26}$ J.M. Moriceau, op. cit. ; É. Baratay, L'Histoire, op. cit., à paraitre ; Maria Iloopoulou, Linda Kaloff, « Cultural history of fighting dog », Une bête parmi les hommes : le chien, op. cit., à paraitre.

${ }^{27}$ C. Beck, E. Fabre, « Interroger le loup historique? Entre la biologie et l'histoire: un dialogue interdisciplinaire », dans J.M. Moriceau \& A. Madeline, éds, Repenser le sauvage grâce au retour du loup. Les sciences humaines réinterrogées, Caen, MSH, 2010: 13-21.

${ }^{28}$ D. Lestel, Les Origines animales de la culture, Paris, Flammarion, 2001; Y. Christen, L'Animal est-il une personne?, Paris: Flammarion, 2009: 261, 267.
} 
coletivos com os seres humanos, aproximam portanto a história, as ciências humanas e as ciências do animal, da etologia à zootecnia, quando se concentra também cada vez mais sobre as atitudes animais, como mostram os trabalhos de Jocelyne Porcher passando pouco a pouco pelo estudo das comunidades criadores-animais, dos primeiros aos segundos ${ }^{29}$.

Se interessar pelo animal em si, estudá-lo de perto, supõe quebrar ao mesmo tempo as barreiras instaladas entre os homens e os animais, entre as ciências humanas e as ciências naturais, entre a ciência histórica e as do presente. Isto requer a colaboração entre especialistas das disciplinas envolvidas como acontece cada vez mais, mas também, e ainda não é generalizado, que cada um se aproprie de conceitos e dados oriundos de outras ciências ${ }^{30}$, e até mesmo que se recomponha para melhores análises. Foi proposto combinar etologia e etnologia para estudar as comunidades homensanimais, onde se compartilham os sentidos, os interesses, os sentimentos, onde se elabora uma sociabilidade interespecífica complexa, e isso significa compreender os animais como a antropóloga Elizabeth Marshall Thomas tentou fazer ${ }^{31}$. Também seria bom cruzar as ciências em uma perspectiva diacrônica, construir, por exemplo, uma história etológica para avaliar a evolução dos comportamentos animais, tais como dos cães passando da errância, ou do trabalho, para a companhia, ou ainda, das vacas passando da polivalência à especialização leiteira e uma etologia histórica para analisar esses comportamentos em um determinado período.

Enquanto isso, o leitor constatará que todas as novas abordagens sócioantropológicas que eu mencionei são muito bem ilustradas nos artigos seguintes ${ }^{32}$. Elas são aqui capitaneadas por um grupo de jovens investigadores ousados, ativos e inventivos, a quem agradeço por terem me associado à presente empreitada, e que nos mostram, como havíamos constatado com André Micoud durante a jornada de estudo que originou este volume, a que ponto as problemáticas sócio-antropológicas mudaram desde a década de 1980, obrigando os seniors que nós nos transformamos à continuar

\footnotetext{
${ }^{29}$ Éleveurs et animaux, réinventer le lien, Paris, PUF, 2002; « L'animal d'élevage n'est pas si bête», Ruralia, 14, 2004: 159-170 ; Une vie de cochon, Paris, La Découverte, 2008.

${ }^{30}$ C. Beck, E. Fabre, art. cit. ; É. Baratay, L'Histoire, op. cit.

31 "Ethology and ethnology: the coming synthesis », Social, science, information, 45, 2006, 2; E. Marshall Thomas, La Vie secrète des chiens. Une anthropologue au pays canin, Paris, Robert Laffont, 1995, et Les Chats et leur culture, Paris, Robert Laffont, 2004. Voir aussi « Les animaux pensent-ils ? », Terrain, 34, 2000.

${ }^{32} \mathrm{O}$ autor refere-se aos artigos do Dossier Relations Anthropozoologiques.
} 
pensando para não nos tornarmos velhos macacos e sermos engolidos por estes jovens lobos. Ah, as vacas!

\section{Referências}

BOBBÉ, S. L'Ours et le loup. Essai d'anthropologie symbolique. Paris: MSH, 2002.

CEGARRA, M. L'animal inventé. Ethnographie d'un bestiaire familier, Paris: L'Harmattan, 2000.

DALLA BERNARDINA, S. L'Utopie de la nature, chasseurs, écologistes et tourists. Paris: Imago, 1998.

Métaillé, 2006. . L'Éloquence des bêtes: quand l'homme parle des animaux. Paris:

DESCOLA, P. Par-delà nature et culture, Paris, Gallimard, 2005.

Ethnologie française, « Les animaux de la discorde », 39 (1), 2009.

GALINON-MÉLÉNEC, B. (éd.), Homme-animal. Quelles relations? Quelles communications? Rouen: PU Rouen, 2003.

GUILLO, D. Des Chiens et des humains. Paris: Pommier, 2009.

HÉRAN, F. "Vers une sociologie des relations avec la nature », Revue Française de Sociologie, 48 (4): 795-806, 2007.

LESCUREUX, N. « Towards the necessity of a new interactive approach integrating ethnology, ecology and ethology in the study of the relationship between Kyrgyz stockbreeders and wolves ». Social, science, information, 45 (3): 463-478, 2006.

MAUZ, I. Gens, cornes et crocs. Versailles: INRA, 2005.

Étienne, 2008.

Les Collectifs et leurs natures. Dossier de synthèse d'HDR, Université de Saint-

MICOUD, A. «Ces bonnes vaches aux yeux si doux ». Communications, 74: 217-237, 2003.

. " Mais qu'ont-ils donc tous à s'occuper des animaux? », In: FRIOUX, S. et

PÉPY. E. A. (éds). L'Animal sauvage entre nuisance et patrimoine. Lyon: ENS Éditions, 2009, p. 177-187.

PIETTE, A. « Entre l'homme et le chien. Pour une ethnographie du fait socio-animal ». Socioanthropologie, 11: 87-104, 2002.

PINÇON-CHARLOT, M. " La place de l'animal dans la société des $\mathrm{XX}^{\mathrm{e}}-\mathrm{XXI}$ siècles ». In:

Collectif, La Recherche médicale à l'aube du XXIe siècle: recherche médicale et modèle animal. Paris: Elsevier, pp. 24-28, 2002.

RÉMY, C. «Une mise à mort industrielle "humaine"? L'abattoir ou l'impossible objectivation des animaux », Politix, 64: 51-73, 2003.

Economica, 2009.

La Fin des bêtes, une ethnographie de la mise à mort des animaux. Paris:

SAUMADE, F. Les Tauromachies européennes. La forme et l'histoire, une approche anthropologique. Paris: CTHS, 1998.

TALIN, C. Anthropologie de l'animal de compagnie. Paris: L'Atelier de l'archer, 2000. Techniques et culture, «Les natures de l'homme », 50, 2008.

TOURRE-MALEN, C. Femmes à cheval, la féminisation des sports et des loisirs équestres : une avancée ? Paris: Belin, 2006. 
Tradução : Manoel Garcia Felix $\mathbf{J r}^{\mathbf{3 3}}$

Revisão: Flávio Leonel Abreu da Silveira ${ }^{34}$

Recebido em: 29/10/2016.

Aprovado em: 06/12/2016.

${ }^{33}$ Universidade Federal do Pará, Brasil.
${ }^{34}$ Universidade Federal do Pará, Brasil. 